\title{
ANALFABETISMO E INSTRUCCIÓN PRIMARIA EN LA PROVINCIA DE BADAJOZ DURANTE EL PRIMER TERCIO DEL SIGLO XX
}

\author{
Cristina Redondo Castro* \\ Francisco Martin Zúñiga**
}

\section{RESUMEN}

En este artículo se analiza la evolución que experimentó la instrucción primaria en la provincia de Badajoz en el primer tercio del siglo XX. En este trabajo hemos recurrido tanto al análisis cuantitativo como al cualitativo, pues nuestro interés se ha centrado en buscar las causas que influyeron en el elevado índice de analfabetismo de la población pacense y su falta de escolarización. De este trabajo se desprende la conclusión de que existe una estrecha relación entre la estructura socioeconómica de un lugar y el grado de instrucción primaria.

Palabras claves: escolarización, alfabetización, analfabetismo, Extremadura, Badajoz.

\footnotetext{
* Universidad de Málaga (UMA), Málaga, Espanha.

*** Universidad de Málaga (UMA), Málaga, Espanha.
} 


\section{ILITERACIA E INSTRUÇÃO PRIMÁRIA NA PROVÍNCIA DE BADAJOZ DURANTE O PRIMEIRO TERCEIRO DO SÉCULO XX}

\section{RESUMO}

Este artigo analisa a evolução do ensino básico na província de Badajoz no primeiro terço do século XX. Neste trabalho recorremos à análise quantitativa e qualitativa, visto que o nosso interesse se centrou em encontrar as causas que influenciaram a elevada taxa de analfabetismo da população de Badajoz e a sua falta de escolaridade. Conclui-se deste trabalho que existe uma estreita relação entre a estrutura socioeconômica de um local e o grau de ensino fundamental.

Palavras-chave: escolaridade, alfabetização, analfabetismo, Extremadura, Badajoz.

\section{ILLITERACY AND PRIMARY INSTRUCTION IN THE PROVINCE OF BADAJOZ DURING THE FIRST THIRD OF THE TWENTIETH CENTURY}

\section{ABSTRACT}

This article analyzes the evolution of primary education in the province of Badajoz in the first third of the 20th century. In this work we have resorted to both quantitative and qualitative analysis, since our interest has focused on finding the causes that influenced the high illiteracy rate of the Badajoz population and their lack of schooling. From this work the conclusion is drawn that there is a close relationship between the socioeconomic structure of a place and the degree of primary education.

Keywords: schooling, literacy, illiteracy, Extremadura, Badajoz.

\section{ILLITÉRATIE ET ENSEIGNEMENT PRIMAIRE DANS LA PROVINCE DE BADAJOZ AU COURS DU PREMIER TROISIÈME SIÈCLE DU XXE SIÈCLETULO EM FRANCÊS}

\section{RESUME}

Cet article analyse l'évolution de l'enseignement primaire dans la province de Badajoz au premier tiers du XXe siècle. Dans ce travail, nous avons eu recours à une analyse à la fois quantitative et qualitative, car notre intérêt s'est concentré sur la recherche des causes qui ont influencé le taux élevé d'analphabétisme de la population de Badajoz et leur manque de scolarité. De ces travaux, on tire la conclusion qu'il existe une relation étroite entre la structure socio-économique d'un lieu et le degré de l'enseignement primaire.

Mots-clés: scolarité, l'alphabétisation, analphabétisme, Extremadura, Badajoz. 


\section{INTRODUCCIÓN}

Son numerosos los trabajos centrados en la temática que nos ocupa. Los investigadores han recuperado y analizado las fuentes historiográficas, a la vez que ofrecidos datos sistematizados. Dentro de dichos trabajos destacaríamos los de Guereña (1988; 2018), Viñao Frago (1984; 1993; 1998; 1999; 2006; 2009), Samaniego Boneu (1973), Hernández Díaz (2000), Luzuriaga (1919), Liébana Collado (2009), Tiana (1987), Martín Zúñiga (1992), Borrás Llop (1996), Vilanova Ribas y Moreno Juliá (1992), etc., los cuales han sido fundamentales para la ejecución de este trabajo.

Gracias a dichos estudios sabemos que superar la plena alfabetización y escolarización en España ha sido un proceso muy largo, lento y desigual. Por ejemplo, en la época que investigamos nos encontramos con provincias como Santander, Álava o Burgos con unos índices de analfabetismo por debajo, incluso, de la media europea en aquellos años, en torno al $12 \%$, mientras que otras provincias, como las andaluzas, canarias y extremeñas, superan el 70\% de iletrados. Precisamente, nuestro estudio se centra en una de las provincias extremeñas, Badajoz, que junto con Cáceres conforma lo que hoy se denomina la Comunidad Autónoma de Extremadura.

Esta provincia tardará muchos años en adquirir la condición de alfabetizada, debido básicamente a unas políticas que no se tomaran en serio la obligatoriedad de la enseñanza de 6 a 12 años establecida en el Real Decreto de 26 de octubre de 1901, máxime cuando en nuestro país la escuela era prácticamente el único instrumento para alfabetizar.

En el caso concreto de Extremadura, gracias a estudios como los de Rodillo Cordero (1998), Cortés Cortés (2016), Domínguez Rodríguez (2005), Jaraíz Cabanillas (2017, 2019), entre otros, conocemos la evolución de la enseñanza en la región, desde mediados del siglo XIX hasta 1940, pero no existen estudios monográficos como el nuestro centrados en la evolución del analfabetismo y la escolarización. No nos limitamos al examen de tablas o 
gráficas que ofrezcan datos con respecto a las diferencias por sexo y edad, nos interesa indagar también en las carencias y ausencias de índole social, cultural y económico que impidieron un desarrollo apropiado de la acción alfabetizadora y propiciaron el alto índice de población en edad escolar que no sabían leer ni escribir.

Antes de observar esa realidad, hemos creído conveniente clarificar, en un primer apartado, aquellos conceptos (analfabetismo, alfabetización, escolarización) que manejaremos a lo largo del estudio. Nos ha sorprendido que la primera vez que aparece oficialmente en nuestro país el concepto de alfabetización es en el diccionario de la Real Academia de 1970, en tanto que la noción de analfabetismo surge mucho antes (1914). Por último, resulta llamativo como el término escolarización ha coincidido con el de alfabetización en nuestra historia de la educación reciente.

En un segundo apartado comenzamos abordando la situación social y económica extremeña de principios del siglo $\mathrm{XX}$, con altas tasas de nacimientos y defunciones, especialmente las asociadas a la mortalidad infantil, fuertes desigualdades sociales y elevado índice de paro provocado por un sistema productivo latifundista, controlado por una oligarquía caciquil minoritaria, que domina el poder económico y político.

Continuamos el artículo hablando del analfabetismo y la escolarización. Demostramos que Extremadura, junto con Andalucía, Canarias, Castilla la Mancha y Murcia forma la denominada España analfabeta, cuyas tasas, en este periodo y mucho después, se mantiene siempre por encima de la media nacional. Esa evidencia es palpable en la provincia de Badajoz, donde en 1930 sólo el 32,6\% del total de la población escolar sabe leer y escribir. No obstante, como ocurre en el resto del país, las estadísticas nos demuestran como en la década de los años veinte hay una mayor reducción de las tasas de iletrados en comparación con las décadas anteriores, debido al incremento importante de escuelas públicas.

Finalmente, conviene resaltar que este trabajo nos ha obligado al 
estudio y consulta de unas fuentes no exentas de dificultad a la hora de extraer e interpretar los datos: falta de correspondencia cronológica entre los censos y estadísticas escolares; desajustes entre la edad escolar real y la legal; imprecisiones en las edades y sexo; falta de censos claros de escuelas privadas y públicas, lo que dificulta la posibilidad de poder establecer resultados globales; con frecuencia, los datos aportados son ofrecidos por los propios implicados, los maestros, y es posible que lo declarado no se correspondan plenamente con la realidad escolar. Pero pese a estas dificultades, hemos encontrado evidencias suficientes que nos ha permitido concluir con éxito nuestra investigación.

\section{CONCEPTUALIZACIÓN DEL PROBLEMA}

"El analfabetismo es consecuencia de la falta de un proceso de alfabetización" (VIÑAO FRAGO, 1984, p. 152). En ese proceso pueden intervenir diversos agentes (familia, iglesia, municipio, etc.), pero si las políticas educativas no saben o no quieren atajar la falta de alfabetización de la población escolar, como sucede en Extremadura y en la provincia de Badajoz durante el periodo investigado, difícilmente se puede mejorar la situación.

Para comprender esa inercia, analizamos la incidencia que puede tener en el proceso de alfabetización variables como diferencias entre el campo y la ciudad, aspectos ideológicos o proselitistas, peculiaridades sociales, el papel de la escuela, aunque como puntualiza Viñao Frago (1984) en este periodo, en nuestro país, todas esas variables no mantuvieron la intensidad y duración suficiente para producir avances significativos, como si sucedió en Inglaterra, Suecia, Prusia o Francia. En términos generales, en estos años asistimos a una alfabetización gradual, lenta y dilatada, que tiene sus inicios a mediados del siglo XIX, al amparo de la Ley Moyano (1857), y que experimenta un impulso importante a partir de los años veinte, debido a factores económicos, el éxodo rural y a la incorporación de la mujer al mundo laboral asalariado (VIÑAO FRAGO, 1999, p. 83-84). 
La acepción de alfabetización como "enseñar a leer y escribir" es relativamente reciente. Surge por primera vez en el diccionario de la Real Academia Española de la lengua de 1970, lo cual demuestra que esta cuestión tenía escasa relevancia social. Sin embargo, el vocablo analfabetismo apareció a principios del siglo XX en el diccionario de 1914: se define analfabeto como "ignorante que ni aún conoce el alfabeto", y en 1925 se cambia por «el que no sabe leer", mientras que analfabetismo sería "la falta de instrucción elemental de un país" (VIÑAO FRAGO, 2009, p. 6). En definitiva, estamos ante un concepto de difícil concreción, dependiendo del entorno sociocultural en el que se plantee e "incluso habría que tener en cuenta la curva evolutiva del concepto referida a los distintos tiempos históricos" (SAMANIEGO BONEU, 1973, p. $375)$.

La España del primer tercio del siglo XX presenta un repartimiento territorial del analfabetismo muy dispar: por ejemplo, en el censo de 1920 nos encontramos con provincias como Santander (10,80\%), Álava (14,50\%) o Burgos (15,30\%) con unos índices por debajo, incluso, de la media europea, frente a otras provincias como Almería (63,28\%), Granada (64,96\%), Jaén (70,65\%) o Badajoz (71,80\%) que tardarán aún muchos años en adquirir la condición de provincias alfabetizadas (MARTÍN ZÚÑIGA, 1993, p. 26; TIANA, 1987, p. 44-45). En ello inciden factores de carácter económico, como el tipo de propiedad de la tierra -en el latifundismo hay mayor número de analfabetos-, el nivel de renta por habitante, el desarrollo comercial y el mayor o menor aislamiento por la deficiente red viaria, a lo que hay que añadir aspectos de carácter cultural, como la incidencia de los "indianos" en la fundación de escuelas en Asturias y Galicia (VIÑAO FRAGO, 2009, p. 11-12).

Todo esto hace sospechar que existen más analfabetos en el campo que en la ciudad, más en las regiones agrícolas pobres que en las ricas e industrializadas, aunque hay que reconocer que no siempre estos parámetros se ajustan a la realidad española de aquellos momentos, pues regiones ricas como Cataluña (25,9\% en 1920) o Valencia (48,05\% en 1920) tienen mayor porcentaje 
de analfabetos que otra relativamente más pobre como Castilla la Vieja (16,8\% en 1920) (MARTÍN ZÚÑIGA, 1993, p. 26; TIANA, 1987, p. 44-45). Por tanto, resulta arriesgado establecer una escala de factores que incidan de un modo determinante, aunque ello no impide que autores como Lorenzo Luzuriaga (1919) consideren que la causa más genérica y decisiva sea la falta de escuelas.

Otro punto polémico es la edad a partir de la cual un sujeto se considera analfabeto. El Real Decreto de 26 de octubre de 1901 y la ley de 23 de junio de 1909 amplían la obligatoriedad de la enseñanza hasta los doce años y, por consiguiente, parece lógico que los mayores de dicha edad que trascurrido el periodo escolar obligatorio no han podido, o no han querido, adquirir el aprendizaje de la lectura y la escritura sean denominados analfabetos. Lo que sucede es que ese dato es difícil de contrastar pues los censos de esos años incluyen dicha edad dentro de un intervalo de entre los once y quince años. Por ese motivo; posiblemente, el criterio que se adoptó en estudios de la época fue el de considerar analfabetos a los sujetos mayores de diez años que no sabían leer y escribir (LUZURIAGA, 1919, p. 1), valor que sí se puede extraer sin dificultad de los mencionados censos de población.

El otro concepto que quisiéramos precisar es el de escolarización, entendida como el acto y el resultado de escolarizar, es decir, en lograr que aquellos y aquellas que están en edad escolar asistan a los centros educativos y completen los estudios que el Estado fija como obligatorios. En España ha coincidido con el de alfabetización, es decir, la escuela ha sido, y es, la principal, a veces la única, "agencia de introducción en el mundo de la cultura escrita" (VIÑAO FRAGO, 2009, p. 12).

Su estudio nos enfrenta con un primer problema, el de las fuentes, pues con frecuencia los datos que aportan dichas fuentes son ofrecidos por los propios implicados, los maestros, y es posible que lo declarado no se corresponda plenamente con la realidad escolar; tampoco hay en estos años unos censos claros de escuelas privadas, por lo que es difícil establecer resultados globales; por último, quienes se encargan de recabar la información 
son, a su vez, los responsables de organizar la enseñanza primaria lo que se puede prestar a cierto "maquillamiento" de los datos (GABRIEL, 1997, p. 228). Por tanto, como afirma Molino (2015, p. 140) "hay que tomar con prevención las estadísticas de los países que tienen muchos problemas", como ocurre con España, que no son capaces de dignificar la vida de sus campesinos o construir carreteras entre sus pueblos o escuelas.

Pero pese a estas dificultades en torno a la fiabilidad de los datos, nos encontramos con otros inconvenientes: falta de correspondencia cronológica entre los censos y las estadísticas escolares; los cambios comentados sobre la edad escolar obligatoria; desajustes entre la edad escolar real y la legal; imprecisiones en el conocimiento real de las edades y sexo del alumnado, pues con frecuencia en los censos estos datos vienen en intervalos de tres o cuatro años (GABRIEL, 1997, p. 228).

En síntesis, los conceptos de analfabetismo, alfabetización y escolarización ofrecen diversos matices en función de la época, contextos..., siendo nuestro objetivo analizarlos dentro del ámbito extremeño, y, más concretamente, en la Badajoz de principios del siglo XX.

\section{LA REALIDAD SOCIAL Y CULTURAL EN LA PROVINCIA DE BADAJOZ: EL PROBLEMA DEL ANALFABETISMO.}

Partimos del convencimiento de que "toda experiencia educativa es, en sí misma, una experiencia social concreta, donde las intervenciones pedagógicas se ven condicionadas por las políticas e ideologías del momento" (MARTÍN ZÚÑIGA, 1993, p. 15). Es por ello que, para analizar la problemática que pretendemos investigar, se hace necesario profundizar en el contexto sociocultural de la región extremeña y pacense de esos momentos.

La imagen sociológica de Extremadura a principios del siglo XX es 
desoladora: elevadas tasas de nacimientos y defunciones, sobre todo por infecciones y parasitismos, siendo las más elevadas las asociadas a la mortalidad infantil; fuerte crecimiento demográfico al recibir población inmigrante que viene a ocupar los puestos que deja la población que emigra a las grandes ciudades en busca de mejores oportunidades; un gran desequilibrio entre los diferentes sectores económicos, con un predominio del sector agrario, donde trabaja el $80 \%$ de la población activa, del que dependen los demás sectores (REY VELASCO, 1998, p. 675).

Baumeister (1997) recoge que en 1877 cuatro quintas partes de la población activa de la provincia de Badajoz estaban ocupadas en la agricultura y la ganadería. El sector secundario y el sector terciario, en cambio, tenían poca importancia con valores en torno al 10\% cada uno. Estos datos, sufrieron pocas modificaciones en los siguientes cuarenta años, donde continuó el excesivo peso del sector agrario. En 1920 todavía las tres cuartas partes de la población activa estaban ocupadas en la agricultura.

Sin embargo, en estos años España, progresivamente, comenzó a dejar de ser un país netamente agrario. Siendo en 1930 cuando se aprecia que más de la mitad de la población trabajaba en los sectores industriales $(26,5 \%)$ o servicios (28\%). Los datos nos muestran el lento trasvase, aún en 1930, del sector agrario $(45,5 \%)$ al industrial, con un ligero incremento en el sector servicios. Estas cifras se contraponen con los datos de las zonas agrarias, como es el caso de Badajoz, donde en 1920 el sector servicios contaba con un 10\%, mientras que la industria y los oficios, con un escaso $13 \%$, habían alcanzado los porcentajes que España tenía en 1877 (BAUMEISTER, 1997).

A pesar de estos datos, la agricultura extremeña a lo largo del siglo XX experimentó una serie de transformaciones que mejoraron la actividad económica de la región. La producción agraria ascendió de 1900 a 1920 en más de un tercio; en 1930 el valor de la producción agraria estaba en un $76 \%$ por encima del de 1900 (BAUMEISTER, 1997). Esta mejoría se debió al aumento de las superficies cultivadas, a una mayor diversificación de cultivos, con 
potenciación o introducción de nuevos productos y a la consiguiente reducción de la extensión ocupada por el pastizal extensivo. Es importante mencionar que esta expansión de la agricultura regional se logró de manera muy rudimentaria.

La producción ganadera estaba muy por detrás de la agraria en sus porcentajes, debido al abandono que sufrió ante la preocupación dominante de la agricultura y especialmente por la obsesión de cultivar las tierras. Pero los datos de los censos ganaderos nos muestran la recuperación y reorientación de estos productos en Extremadura y, pese a ese abandono sufrido, el incremento fue más espectacular que las tasas de producción agrícola (GARCÍA, SÁNCHEZ Y MERINERO, 1985, p. 916-918). Por tanto, estamos ante una región que se caracteriza por un profundo retraso económico y fuertes desigualdades sociales que desencadenan diversos conflictos. Dichas desigualdades encuentran su origen en el fuerte arraigo del latifundismo y elevado índice de paro y subempleo, lo que provoca la división de la sociedad entre una oligarquía, que aunque minoritaria es la que ostentaba el poder económico y político, y una gran masa proletaria. Durante el reinado de Alfonso XIII, la indignación de braceros y campesinos desemboca en numerosas huelgas y disturbios violentos debidos a la situación insostenible que están viviendo, al no poder alimentar a sus familias por sus bajos jornales y por el encarecimiento de los productos alimentarios. Todo ello viene promovido por un modelo político, el caciquismo, "el cual se encontraba más interesado por favorecer a su 'clientela' que en ofrecer alternativas globales a los problemas de injusticia, miseria e incultura tan arraigados en este país" (MARTÍN ZÚÑIGA, 1993, p. 16-17).

Ese retraso económico, social y cultural trae otro "mal", el analfabetismo. Es cierto que en estos momentos la educación popular, que había estado en un segundo plano, pasa a ser una de las grandes preocupaciones de intelectuales y políticos, quienes reclaman al Estado la implantación de la misma y exigen el derecho y el deber de proporcionar a los ciudadanos una instrucción adecuada, aunque desgraciadamente ese deseo está aún lejos de cumplirse en Extremadura. Basta con recordar que ésta, junto con Andalucía, 
Canarias, Castilla-La Mancha y Murcia forman parte de la denominada España analfabeta, cuyas tasas, en este periodo y mucho después, se mantienen siempre por encima de la media nacional (VILANOVA RIBAS; MORENO JULIÁ, 1992, p. 79).

Para tomar con perspectiva el análisis de la evolución del analfabetismo y sus causas en Badajoz debemos conocer cómo era la situación del mismo en la segunda mitad del siglo XIX. Los datos generales de analfabetismo de la segunda mitad del siglo XIX en la región extremeña muestran una situación preocupante, sin embargo, como recogen Jaraíz Cabanillas et al. (2017, p. 1314), "los estudios realizados sobre este periodo muestran que la situación de las provincias extremeñas en relación con el conjunto de provincias españolas presenta unos valores medios, situándose en los puestos 22 y 23 dentro del conjunto de todas”. Por tanto, podríamos decir que, en este periodo la realidad escolar extremeña sigue los mismos parámetros de instrucción pública e índices de analfabetismo que el conjunto del país. El estudio de Jaraíz Cabanillas et al. (2017), además muestra que existe una distribución desigual por provincias, donde los datos de Badajoz son más acusados que los de Cáceres. En la provincia de Badajoz casi todos los partidos judiciales se encuentran con unos valores de analfabetismos situados entre el 60 y el 90\% y donde el índice de analfabetismo femenino es muy superior al masculino. En 1860, la provincia de Cáceres presenta unos índices de analfabetismo masculino del 62\% mientras la provincia de Badajoz del 70\%, esta desigualdad se mantiene en 1887, que, aunque en las dos provincias hay un descenso de tres puntos (respectivamente, al $59 \% \mathrm{y}$ al $67 \%$ ) las diferencias entre ambas son notables. En cuanto al analfabetismo femenino, en 1860, en la mayoría de los partidos judiciales de Badajoz el panorama es desolador, pues los porcentajes se sitúan por encima del 90\%. Sin embargo, las diferencias comentadas entre las provincias, aunque se dan también entre la población femenina, son menos acusadas. En 1860 el analfabetismo femenino en Cáceres es del 82\% y en Badajoz del 84\%, es decir, hay una diferencia de dos puntos entre ambas provincias, los cuales se reducen a uno en 1887 (69\% para Cáceres y 70\% para Badajoz) y en 1900 (75\% para las 
cacereñas y $76 \%$ para las pacenses). Estos datos llaman nuestra atención, pues se da un retroceso en ambas provincias en cuanto a los índices de alfabetización femenina.

Esta situación no fue mucho más tranquilizadora al inicio del nuevo siglo, pues los datos nos muestran que un $74,1 \%$ de la población extremeña era analfabeta en 1900 , dato superior a la media nacional cifrada en un $63,8 \%$ en esa misma fecha, y que en 1930 descendía a un 55,5\% mientras que en el resto del país bajaba al 44\% (REDONDO CASTRO, 2018, p. 3; VIÑAO FRAGO, 1994, p. 669; JARAÍZ CABANILLAS et al., 2019; BORRÁS LLOP, 1996, p. 352-357), siendo la provincia de Badajoz la que ofrecía un mayor aporte de sujetos iletrados: en 1900 la población pacense analfabeta era el 75\% y desciende al 60\% en 1930 (véase tabla 1), porcentaje que sigue siendo muy elevado y no augura una pronta solución del problema. Mientras tanto, en Cáceres las cifras en las mismas fechas eran menos abultadas, del $71 \%$ a $48 \%$ respectivamente (REDONDO CASTRO, 2018, p. 47).

Tabla 1 - Evolución de analfabetismo en Extremadura (1900-1940)

\begin{tabular}{|l|l|l|l|l|l|}
\hline \multicolumn{1}{|c|}{ AÑO } & \multicolumn{1}{|c|}{$\begin{array}{c}\text { NO DE }^{\text {HABITANTES }} \\
\text { HAMBRES }\end{array}$} & MUJERES & \multicolumn{1}{|c|}{ TOTAL } & \multicolumn{1}{c|}{$\%$} \\
\hline 1900 & 882.410 & 297.814 & 356.330 & 654.144 & 74,1 \\
\hline 1910 & 990.991 & 308.414 & 369.295 & 677.709 & 68,4 \\
\hline 1920 & 1.054 .657 & 308.814 & 377.009 & 685.823 & 65,0 \\
\hline 1930 & 1.152 .172 & 280.200 & 359.409 & 639.609 & 55,5 \\
\hline 1940 & 1.253 .924 & 248.833 & 341.454 & 590.287 & 47,1 \\
\hline
\end{tabular}

Fuente: RODILLO Cordero, Francisco Javier. Datos para la historia escolar de Extremadura. Mérida: Editora regional de Extremadura, 1998. p. 106. 
Tabla 2 - Distribución del analfabetismo por provincias (1900-1930)

BADAJOZ

\begin{tabular}{|l|c|c|c|c|c|c|c|c|c|}
\hline & \multicolumn{3}{|c|}{ POBLACIÓN DE “HECHO” } & \multicolumn{7}{|c|}{ POBLACIÓN ANALFABETA } \\
\hline Año & H & M & T & H & \% & M & \% & T & \% \\
\hline 1900 & 262.685 & 257.561 & 520.246 & 185.142 & 71 & 206447 & 80 & 391.589 & 75 \\
\hline 1910 & 298.193 & 295.013 & 593.206 & 191.125 & 60 & 219.074 & 74 & 410.199 & 69 \\
\hline 1920 & 324.232 & 321.426 & 644.625 & 200.086 & 62 & 230.868 & 72 & 430.954 & 67 \\
\hline 1930 & 350.954 & 351.464 & 702.416 & 179.442 & 51 & 220.003 & 63 & 399.445 & 60 \\
\hline
\end{tabular}

\section{CÁCERES}

\begin{tabular}{|c|c|c|c|c|c|c|c|c|c|}
\hline & \multicolumn{3}{|c|}{ POBLACIÓN DE “HECHO” } & \multicolumn{7}{|c|}{ POBLACIÓN ANALFABETA } \\
\hline Año & H & M & T & H & \% & M & \% & T & \% \\
\hline 1900 & 181.339 & 180.825 & 632.164 & 106.361 & 59 & 139.808 & 77 & 246.169 & 71 \\
\hline 1910 & 197.196 & 200.589 & 397.785 & 117.289 & 59.5 & 150.221 & 75 & 267.510 & 67 \\
\hline 1920 & 202.252 & 207.780 & 410.032 & 108.728 & 53.8 & 146.141 & 70 & 254.869 & 62 \\
\hline 1930 & 222.585 & 226.240 & 449.756 & 100.758 & 45.3 & 139.406 & 61 & 240.164 & 53 \\
\hline 1940 & 249.206 & 262.171 & 511.377 & 97.791 & 39.2 & 144.889 & 55 & 242.680 & 48 \\
\hline
\end{tabular}

Fuente: Elaboración propia a partir de los datos obtenidos del INE. Anuarios Estadísticos de 1900 a 1920/ Anuario 1912 y 1920; de 1921 a 1930/ Anuario 1930; de 1940 a 1951/Anuario1943;

y Censos de Población.

La dinámica del analfabetismo en la provincia de Badajoz es similar a lo que sucede en otras partes del país, es decir, en el entorno rural las posibilidades de alfabetización son menores en comparación con el medio urbano y ello se debe, como recoge Hernández Díaz (2000, p. 116), a que la decisión técnica de introducir la enseñanza graduada a principios de siglo sólo fue propicio para las ciudades donde se avanzó en las metodologías y en los procesos de aprendizaje de los niños pero, sin embargo, a la escuela de las zonas rurales le hizo un flaco favor, dado que anulaba la personalidad de las mismas y las dejaba en desventaja respecto al modelo dominante de escuela urbana. En la tabla 3 podemos ver como se refleja con claridad esta situación, donde se aprecia que en Badajoz capital se mantienen unos índices bastante más bajos que en el resto de partidos judiciales: en 1900 es el 60,2\%, mientras que a nivel 
provincial sube al 75,3\%, y en 1930 baja al 46,8\% frente al 59,9\% respectivamente. La distribución provincial en esos años de quienes no saben leer y escribir tiende a cierta homogenización, pues los porcentajes entre los partidos judiciales son similares, con una diferencia entre el mayor y el menor de unos nueve puntos como máximo: por ejemplo, en 1900 esta entre el 71,7\% de Olivenza y el 79,4\% de Villanueva de la Serena, en tanto que en 1930 va del 52,5\% de Mérida al el 63,5\% de Herrera del Duque (ver tabla 3). También se observa, de modo generalizado, como en la década de los años veinte hay una mayor reducción de las tasas de iletrados en comparación con las décadas anteriores debido, como luego comentaremos, al incremento importante de escuelas públicas. Sin embargo, los partidos judiciales de Don Benito, Herrara del Duque y Villafranca presentan un retroceso alfabetizador entre 1910 y 1920, si bien esta situación se corrige en la década siguiente con aumentos más o menos continuados en todos los partidos.

Tabla 3 - Evolución del analfabetismo en Badajoz por partidos judiciales (1900-1930)

\begin{tabular}{|c|c|c|c|c|c|c|c|c|c|c|c|c|}
\hline \multirow[b]{2}{*}{$\begin{array}{l}\text { PARTI } \\
\text { DOS } \\
\text { JUDICI } \\
\text { ALES }\end{array}$} & \multicolumn{3}{|c|}{1900} & \multicolumn{3}{|c|}{1910} & \multicolumn{3}{|c|}{1920} & \multicolumn{3}{|c|}{1930} \\
\hline & 量 & $\%$ & 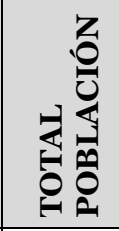 & 空 & $\%$ & 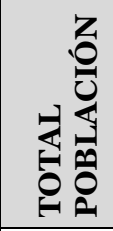 & 究。 & $\%$ & 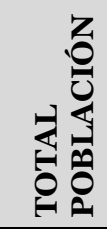 & 量 & $\%$ & 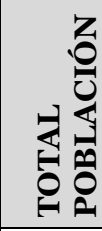 \\
\hline $\begin{array}{l}\text { Alburque } \\
\text { que }\end{array}$ & 18021 & 75,8 & 23775 & $\begin{array}{l}2290 \\
7\end{array}$ & 75,9 & 30163 & 21565 & 72,4 & 29804 & 20080 & 61,8 & 32501 \\
\hline $\begin{array}{l}\text { Almendr } \\
\text { alejo }\end{array}$ & 37708 & 76,8 & 49090 & 37991 & 68,2 & 55703 & 38587 & 66,3 & 58183 & 39312 & 58,1 & 67716 \\
\hline Badajoz & 20629 & 60,2 & 34286 & $\begin{array}{l}2102 \\
6 \\
\end{array}$ & 54,2 & 38814 & 21474 & 50,2 & 42810 & 23346 & 46,8 & 49854 \\
\hline Castuera & 29231 & 77,7 & 37607 & 34615 & 74,4 & 46499 & 37055 & 72,5 & 51124 & 34843 & 59,6 & 58435 \\
\hline $\begin{array}{l}\text { Don } \\
\text { Benito }\end{array}$ & 22467 & 75,5 & 29735 & $\begin{array}{l}2158 \\
4 \\
\end{array}$ & 63,0 & 34265 & 25165 & 66,7 & 37725 & 21112 & 53,7 & 39341 \\
\hline $\begin{array}{l}\text { Fregenal } \\
\text { de la } \\
\text { Sierra }\end{array}$ & 27730 & 78,8 & 35186 & $\begin{array}{l}2738 \\
3\end{array}$ & 71,1 & 38489 & 27394 & 67,2 & 40774 & 18157 & 53,9 & 33663 \\
\hline $\begin{array}{l}\text { Fuente } \\
\text { de } \\
\text { Cantos }\end{array}$ & 28208 & 78,9 & 35734 & $\begin{array}{l}2809 \\
0\end{array}$ & 70,1 & 40078 & 28166 & 63,2 & $4455^{\circ}$ & 27754 & 60,5 & 45898 \\
\hline
\end{tabular}




\begin{tabular}{|c|c|c|c|c|c|c|c|c|c|c|c|c|}
\hline \multirow[b]{2}{*}{$\begin{array}{l}\text { PARTI } \\
\text { DOS } \\
\text { JUDICI } \\
\text { ALES }\end{array}$} & \multicolumn{3}{|c|}{1900} & \multicolumn{3}{|c|}{1910} & \multicolumn{3}{|c|}{1920} & \multicolumn{3}{|c|}{1930} \\
\hline & 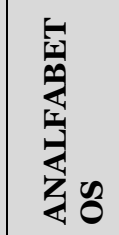 & $\%$ & 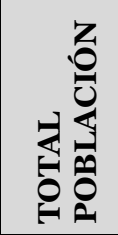 & 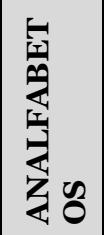 & $\%$ & 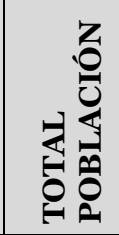 & 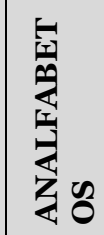 & $\%$ & 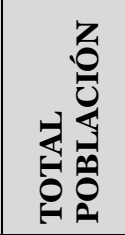 & 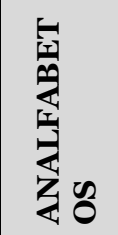 & $\%$ & 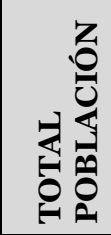 \\
\hline $\begin{array}{l}\text { Herrera } \\
\text { del } \\
\text { Duque }\end{array}$ & 17069 & 79,0 & 21610 & 17674 & 72,3 & 24451 & 19485 & 74,9 & 26026 & 19092 & 63,5 & 30088 \\
\hline $\begin{array}{l}\text { Jerez de } \\
\text { los } \\
\text { Caballer } \\
\text { os }\end{array}$ & 30256 & 78,6 & 38484 & \begin{tabular}{|l}
2982 \\
o
\end{tabular} & 72,6 & 41045 & $\begin{array}{l}3386 \\
2\end{array}$ & 69,1 & 48975 & 30522 & 58,8 & 51932 \\
\hline Llerena & 41282 & 77,9 & 52972 & $\begin{array}{l}3954 \\
8\end{array}$ & 68,2 & 57966 & $\begin{array}{l}4402 \\
6\end{array}$ & 67,3 & 65399 & 39186 & 56,0 & 69946 \\
\hline Mérida & 34607 & 71,8 & 48209 & 35711 & 62,5 & 57143 & 37310 & 60,0 & 62134 & 37467 & 52,5 & 71340 \\
\hline Olivenza & 22405 & 71,7 & 31241 & \begin{tabular}{|l|}
2594 \\
$\mathrm{o}$ \\
\end{tabular} & 70,7 & 36685 & 24114 & 62,8 & 38400 & 22482 & 55,2 & 40726 \\
\hline Alcocer & 15456 & 74,9 & 20647 & 16123 & 68,2 & 23640 & 16759 & 66,5 & 25211 & 16223 & 57,7 & 28107 \\
\hline $\begin{array}{l}\text { Villanue } \\
\text { va de la } \\
\text { Serena }\end{array}$ & 22053 & 79,4 & 27764 & \begin{tabular}{|l|}
2369 \\
3
\end{tabular} & 73,2 & 32382 & 23712 & 73,2 & 32373 & 19450 & 56,1 & 34702 \\
\hline Zafra & 24273 & 72,0 & 33692 & $\begin{array}{l}2318 \\
\mathrm{o}\end{array}$ & 64,7 & 35849 & 43651 & 77,2 & 56564 & 25581 & 54,2 & 47199 \\
\hline TOTAL & \begin{tabular}{|l|}
39139 \\
5
\end{tabular} & 75,2 & $\begin{array}{l}5^{2003} \\
2\end{array}$ & \begin{tabular}{|l|}
4052 \\
85
\end{tabular} & 68,3 & $\begin{array}{l}\mathbf{5 9 3 1 7} \\
\mathbf{2}\end{array}$ & \begin{tabular}{|l|}
4423 \\
25
\end{tabular} & 67,0 & \begin{tabular}{|l|l}
66005 \\
2
\end{tabular} & $\begin{array}{l}39460 \\
7\end{array}$ & 56,3 & \begin{tabular}{|l|}
70144 \\
8
\end{tabular} \\
\hline
\end{tabular}

Fuente: Elaboración propia a partir de los datos obtenidos de los Censos de población. Clasificación de la población de HECHO por sexo, estado civil e instrucción elemental. Tomo III. Volúmenes regionales. Fondo documental del Instituto Nacional de Estadística.

De todos modos, los porcentajes siguen siendo muy altos y si nos referimos al caso de las mujeres la situación es aún más vergonzante. En los inicios de siglo XX todavía era escaso el interés, desde amplios sectores de la sociedad y la política del país, por la escolarizar plena de aquéllas, y Badajoz, en ese sentido, no fue una excepción. Prueba de ello es que en 1900 nos encontramos con que el 80,1\% del total eran analfabetas en tanto que los varones analfabetos suponían el 69,5\%, y treinta años después, aunque se produce un descenso importante, todavía un 61,9\% de mujeres no saben leer ni escribir frente al 50,6\% en el caso de los varones (véase tabla 4). Es evidente que si los datos los desglosamos por partidos judiciales, el de Badajoz presenta unos 
porcentajes de mujeres $(65,8 \%$ en 1900 y $52,5 \%$ en 1930$)$ y hombres $(55,1 \%$ en 1900 y $42 \%$ en 1930) analfabetos bastante inferiores a los registrados en el resto de la provincia, donde hay partidos judiciales, como el de Herrera del Duque, en el que las mujeres marcaron índices superiores al 80\% en las tres décadas.

Tabla 4 - Evolución del analfabetismo por género (1900-1930)

\begin{tabular}{|l|l|l|l|l|l|l|l|l|}
\hline \multirow{2}{*}{ PARTIDOS JUDICIALES } & \multicolumn{2}{|c|}{ 1900 } & \multicolumn{2}{c|}{ 1910 } & \multicolumn{2}{c|}{1920} & \multicolumn{2}{c|}{1930} \\
\cline { 2 - 10 } & H\% & M\% & H\% & M\% & H\% & M\% & H\% & M\% \\
\hline Alburqueque & 69,4 & 82,5 & 69,9 & 82,0 & 68,5 & 76,2 & 55,5 & 68,1 \\
\hline Almendralejo & 72,6 & 81,1 & 64,3 & 72,0 & 59,5 & 72,4 & 53,1 & 62,8 \\
\hline Badajoz & 55,1 & 65,8 & 47,9 & 60,9 & 44,4 & 56,4 & 42,0 & 51,5 \\
\hline Castuera & 71,4 & 83,9 & 69,1 & 79,8 & 67,4 & 77,5 & 53,6 & 65,7 \\
\hline Don Benito & 70,4 & 80,7 & 58,2 & 67,7 & 59,5 & 73,7 & 46,1 & 60,9 \\
\hline Fregenal de la Sierra & 76,2 & 81,5 & 67,0 & 75,6 & 64,5 & 69,9 & 50,6 & 57,2 \\
\hline Fuente de Cantos & 74,9 & 83,1 & 65,4 & 74,8 & 60,1 & 66,4 & 55,6 & 65,4 \\
\hline Herrera del Duque & 72,0 & 86,1 & 64,5 & 80,2 & 67,5 & 82,3 & 55,0 & 72,0 \\
\hline Jerez de los Caballeros & 75,5 & 81,9 & 69,6 & 75,8 & 66,1 & 72,3 & 55,2 & 62,6 \\
\hline Llerena & 73,1 & 82,9 & 63,8 & 72,5 & 61,3 & 73,4 & 50,5 & 61,4 \\
\hline Mérida & 66,8 & 76,9 & 56,6 & 68,6 & 54,0 & 66,3 & 46,6 & 58,5 \\
\hline Olivenza & 68,4 & 75,1 & 66,5 & 75,1 & 58,2 & 67,6 & 50,0 & 60,5 \\
\hline Alcocer & 65,5 & 84,3 & 59,9 & 76,6 & 57,7 & 75,2 & 48,4 & 67,2 \\
\hline Villanueva de la Serena & 74,1 & 84,5 & 69,0 & 77,2 & 67,3 & 79,0 & 48,1 & 63,6 \\
\hline Zafra & 69,3 & 74,8 & 62,1 & 67,2 & 63,2 & 84,5 & 49,7 & 58,7 \\
\hline TOTAL & $\mathbf{6 9 , 5}$ & $\mathbf{8 0 , 1}$ & $\mathbf{6 3 , 4}$ & 73,3 & $\mathbf{6 0 , 8}$ & 72,9 & $\mathbf{5 0 , 6}$ & $\mathbf{6 1 , 9}$ \\
\hline
\end{tabular}

Fuente: Elaboración propia a partir de los datos obtenidos de los Censos de población. Clasificación de la población de HECHO por sexo, estado civil e instrucción elemental. Tomo III. Volúmenes regionales. Fondo documental del Instituto Nacional de Estadística.

Pero hay otro dato que refleja una perspectiva aún más desoladora en la 
evolución de este mal endémico y que explica por qué Extremadura, junto con otras comunidades como Andalucía, Canarias, Murcia, etc., tardará muchas décadas en adquirir la condición de región alfabetizada. Nos referimos, concretamente, a los bajos niveles de alfabetización de la población en edad escolar en la provincia de Badajoz, tal como se aprecia en las siguientes tablas (véase tabla 5). Resulta desolador descubrir que aún en 1930 el 67,4\% de la población entre 6 y 10 años no sabía leer ni escribir (ver tabla 5), o lo que es lo mismo, sólo al 32,6\% podemos considerarla alfabetizada.

A los seis años, edad con la que se inicia la escolarización, el analfabetismo roza el cien por cien en 1900, lo que demuestra, como comentábamos anteriormente, que la escuela era el único ámbito desde donde se iniciaba la alfabetización, situación que apenas cambia al final del tercio pues sólo se baja al 86,6\% (ver tabla 5). Sin embargo, en este periodo se puede hablar de un breve repunte en la escolarización y alfabetización de esta provincia, pues en 1930 el 50\% de quienes tenían diez años sabían, no sabemos en qué grado, leer y escribir, porcentaje que en 1900 sólo era del 22\% (datos extraídos a partir de la tabla 5).

Tabla 5 - Población en edad escolar que no sabe ni leer ni escribir en la provincia de Badajoz (1900-1930)

\begin{tabular}{|c|c|c|c|c|c|c|c|c|}
\hline \multirow[b]{2}{*}{ 旁 } & \multicolumn{2}{|l|}{1900} & \multicolumn{2}{|l|}{1910} & \multicolumn{2}{|l|}{1920} & \multicolumn{2}{|l|}{1930} \\
\hline & 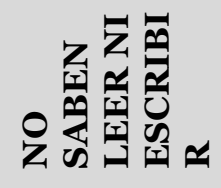 & $\%$ & 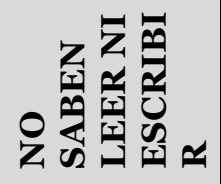 & $\%$ & 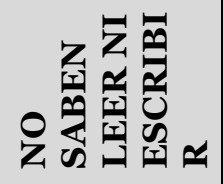 & $\%$ & 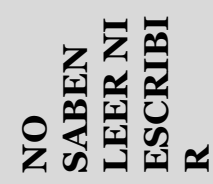 & $\%$ \\
\hline 6 años & 10.860 & 95,0 & 10.440 & 76,8 & 13.148 & 91,7 & 13.589 & 86,6 \\
\hline 7 años & 10.335 & 90,3 & 8.973 & 66,9 & 12.017 & 85,4 & 11.571 & 71,7 \\
\hline 8 años & 10.019 & 84,8 & 8.439 & 61,4 & 12.000 & 79,4 & 9.419 & 63,7 \\
\hline 9 años & 8.753 & 80,9 & 6.715 & 55,5 & 10.189 & 72,9 & 7.892 & 54,3 \\
\hline 10 años & 9.132 & 77,7 & 6.517 & 53,2 & 10.763 & 68 & 7.482 & 49,6 \\
\hline Total & 49.099 & 85,7 & 41.084 & 63,2 & 58.117 & 79,4 & 49.953 & 67,4 \\
\hline
\end{tabular}


Fuente: Elaboración propia a partir de los datos obtenidos de los Censos de población, Badajoz. Tomo III. Clasificación de los habitantes por su edad, combinada por su sexo, estado civil e instrucción elemental. Fondo documental del Instituto Nacional de Estadística.

Otro dato cuantitativo interesante es que apenas existen diferencias porcentuales entre niñas y niños, de entre seis a diez años, en lo que respecta a la falta de los rudimentos en lectura y escritura (ver tabla 6). Ello puede entenderse como que a lo largo de estos años se está produciendo una incipiente tendencia en la reducción de la tradicional supremacía numérica de mujeres analfabetas en comparación con los varones, al menos en lo que a edad escolar se refiere.

Tabla 6 - Población clasificada por género y tanto por ciento que no sabe ni leer ni escribir en la provincia de Badajoz (1900-1930)

\begin{tabular}{|l|l|l|l|l|l|l|l|l|}
\hline \multirow{2}{*}{ EDAD } & \multicolumn{2}{|c|}{ 1900 } & \multicolumn{2}{c|}{ 1910 } & \multicolumn{2}{c|}{ 1920 } & \multicolumn{2}{c|}{ 1930 } \\
\cline { 2 - 9 } & \multicolumn{1}{|c|}{ H\% } & \multicolumn{1}{|c|}{ M\% } & \multicolumn{1}{|c|}{ H\% } & M\% & H\% & M\% & H\% & \multicolumn{1}{|c|}{ M\% } \\
\hline 6 años & 47,9 & 47,2 & 38,3 & 38,5 & 46,3 & 45,4 & 42,9 & 44,4 \\
\hline 7 años & 45,2 & 45,1 & 33,2 & 33,8 & 42,4 & 43,0 & 36,5 & 37,6 \\
\hline 8 años & 42,7 & 42,1 & 31,0 & 30,4 & 40,1 & 39,3 & 34,5 & 31,3 \\
\hline 9 años & 40,6 & 40,3 & 27,8 & 27,7 & 36,1 & 36,8 & 27,9 & 28,1 \\
\hline 10 años & 38,3 & 39,5 & 26,7 & 26,6 & 33,6 & 34,4 & 25,1 & 26,3 \\
\hline Total & 42,9 & 42,8 & 31,6 & 31,6 & 39,6 & 39,7 & 33,6 & 33,8 \\
\hline
\end{tabular}

Fuente: Elaboración propia a partir de los datos obtenidos de los Censos de población, Badajoz. Tomo III. Clasificación de los habitantes por su edad, combinada por su sexo, estado civil e instrucción elemental. Fondo documental del Instituto Nacional de Estadística.

Pero, ¿a qué se debe este elevado índice de analfabetismo? En el caso de Extremadura, Rodillo Cordero (1998) manifiesta que se debió al insuficiente número de escuelas, recordemos que ello ya lo planteaba Lorenzo Luzuriaga como una causa general, pero aquellas no se construían solas, era la sociedad quien debía exigir y a los políticos a quienes les incumbía aprobar los presupuestos necesarios para resolver el problema, pero dicha simbiosis no se 
produjo con la suficiente contundencia. No obstante, hay que reconocer que en este periodo se está iniciando a nivel social e intelectual un cierto debate por las malas condiciones de la enseñanza primaria en España, lo que favorece que a lo largo del primer tercio del siglo $\mathrm{XX}$, especialmente en los años veinte, se produzca un incremento importante, impulsado en gran medida por políticas municipales, en la construcción de escuelas, aunque siguió siendo insuficiente para cubrir las necesidades de toda la población escolar (MARTIN ZÚÑIGA; SANCHIDRIÁN BLANCO, 2012 y 2016).

Los primeros datos sobre el número de escuelas los encontramos en el Anuario del Instituto Nacional de Estadística de 1908. Se especifica que en la provincia de Badajoz había un total de 489 escuelas, de las cuales 401 eran unitarias públicas y 88 privadas, siendo la población en edad escolar de 58.724 personas y la asistencia de 34.092 niños y niñas, donde el $76,6 \%$ iban a las escuelas públicas y el 38,3\% a las privadas. En el Anuario de 1923 el número de escuelas públicas aumenta a 554, de las cuales 549 eran unitarias y 3 graduadas de niños y 2 de niñas. La población en edad escolar estaba censada en 86.367 de los cuales estaban matriculados 39.996, es decir que más de un 50\% no asistía a la escuela.

El problema de la asistencia en Olivenza, no está muy agudizado, [...], y sin embargo, se nota más principalmente en los meses cruciales de invierno y en las épocas en las que las faenas del campo exigen la prestación familiar al obrero. Por muy severas que sean las leyes, y por muy frecuentes que sean las circunstancias y los bandos de gobernadores y alcaldes, por conveniente que sean los razonamientos que inspectores y maestros dirijan a los padres, ni unos ni otros conseguirán que los niños asistan con asiduidad a las escuelas en esas épocas de trabajo rural y esas otras de crudeza invernal, sin vestidos, sin calzado, sin alimentos en sus estómagos.

[...] mientras el hambre se enrosque como serpiente, en las familias pobres, será un imposible la asistencia escolar en las escuelas (ARAGONÉS, 1928, p. 9).

Por tanto, el absentismo era la segunda causa del elevado índice de 
analfabetismo, aunque conviene precisar que el origen de dicho absentismo no fue solo la falta de motivación del alumnado ante unas enseñanzas tediosas impartidas, en muchas ocasiones, en locales inmundos, sino que Badajoz no tenía las escuelas necesarias, ni públicas ni privadas, para acoger a toda su población escolar (REDONDO CASTRO, GRANA GIL Y SANCHIDRIÁN BLANCO, 2017; RODILLO CORDERO, 1998), si nos atenemos a los datos manifestados anteriormente, había en torno a una escuela por cada 122 escolares en 1908 y 150 en 1923. No obstante, hay que reconocer que se incrementó el número de construcciones escolares, llegando en 1930 a 738 y, con ellas, el número de maestros, sin embargo, para haber podido atender a las demandas de instrucción primaria hubiera sido necesario contar con 1.329 escuelas, tal y como afirma Samaniego Boneu (1976, p. 282). Pero no sólo la falta de motivación o de escuelas era la causa de la no asistencia a las mismas, sino que a principios de siglo todavía había miles de niños que sólo asistían a las escuelas por temporadas, pues, debían acudir al campo, donde eran explotados, a trabajar (TIANA, 1987; BORRAS, 1996; VIÑAO FRAGO, 1998; HERNÁNDEZ DÍAZ, 2000 y GARMENDIA; ZABALETA; MURUA, 2018).

Esta falta de escuelas es denunciada en el Correo Extremeño por Antonio Bartolomé Aragonés, quién al referirse a la situación de la enseñanza primaria en el pueblo extremeño de Olivenza denuncia:

Del abandono, de la incuria a que hace tiempo se tenía condenada a la escuela nacional, participaban también las escuelas de la localidad, que con sus 10.000 almas, en el censo escolar, de más de 800 escolares de ambos sexos, sólo contaba con tres escuelas de niños; dos de niñas y una de párvulos, instaladas pésimamente y falta de apoyo moral y material.

[...] Salta a la vista la necesidad, con sólo citar el censo escolar de 900 alumnos. A las escuelas existentes concurren el máximo de matrícula de las mismas, que es el siguiente: a las dos escuelas de niñas con una auxiliar municipal, cada una 200; a la de párvulos con dos auxiliares municipales, 150; a las tres de niños en régimen graduado, 170; total 520, o sea, 380 niños de ambos sexos, que necesitan escuela y no pueden alcanzarla gratuitamente (ARAGONÉS, 1928, p. 9). 
A la cuestión anterior había que añadir las malas condiciones higiénicas y pedagógicas de los locales destinados a escuelas, mal endémico en muchos lugares del territorio nacional. En este sentido el periodista Joaquín Dicenta escribía en 1901 en La Región Extremeña, Diario Republicano:

¡Reformar la enseñanza pública! A eso tira el conde Romanones de una parte y los asambleístas de otra.

iReformar la enseñanza pública!... No es mala idea; solo que reformarla resulta poco. Revolucionarla sería mejor.

No caben reformas en edificios agrietados desde los cimientos hasta el remate. Hace falta echar por tierra el edificio y aprovechar los terrenos libres, para construir otro.

Ruinoso, dislocado, próximo á derrumbarse, aplastando la escasa cultura que nos respeta, está en España el edificio de la Instrucción Pública.

Sí: es necesario que no sean las escuelas de primeras letras sobre un cartel palabras cuyo significado no entienden y canturrean á coro cifras aritméticas y oraciones vacías que para nada sirven; [...] (DICENTA, 1901).

Otro ejemplo de ello es la descripción que Bohoyo Velázquez hace de la situación de la localidad pacense de Montijo en 1902:

Las escuelas Municipales de niñas se hallan instalada en una casa particular [...] la planta baja, que tiene habitaciones espaciosas la ocupa la profesora con su familia, y la escuela radica en los graneros [...] Se encuentra a teja vana, siendo la techumbre tan baja, que por la parte de la calle se da en ella la mano; por ventilación tiene tres huecos bastantes reducidos, todos a la parte norte. El número de niñas es de 238 [...]. Con arreglo al número de habitantes que cuenta esta población, debieran existir, cuando menos, cuatro escuelas de instrucción primaria, sostenidas con fondos públicos”.

Luis Bello Tompreta (1927), en su obra Viaje por las escuelas de España, no duda en calificar las extremeñas como las peores del país, debido a las lamentables condiciones en las que se encontraban, "no hay ni una sola 
escuela digna de este nombre" (p. 22). Es tal la situación que la prensa local se hace eco de ello. Un ejemplo lo tenemos en el Correo Extremeño donde un estudiante de magisterio expresa que:

\begin{abstract}
En este pueblo es preciso construir nuevos locales para las Escuelas Públicas, y dotar todas las existentes de moderno material pedagógico para cumplir sus fines.

Soy alumno del Magisterio y me encuentro realizando las prácticas de enseñanza en la escuela de niños, núm. 2 [...] trabajo en la confección de la Memoria de dichas prácticas, y un profundo sentimiento me va embargando poco a poco en el trascurso de su elaboración. Dicha escuela a pesar de ser la de mejor local de la población, tiene mediana situación, mala orientación, poca superficie; pero cuando más hondamente me he afectado, ha sido al llegar al mueblaje: horrible decepción. [...] Los niños de sus escuelas no disponen para sentarse, más que de pocas, muy pocas bancas higiénicas (doce asientos por escuelas); las demás, son de las antiguas mesas multipersonales [...] (RODRÍGUEZ BOOTELLO, 1928, p. 4).
\end{abstract}

Además, este hecho se veía agravado por las insuficientes dotaciones oficiales a los maestros, por no decir abandono, en cuanto a salario, formación y estabilidad. A pesar de que el Ministerio de Instrucción Pública y Bellas Artes se hizo cargo del pago de los salarios del magisterio público a partir de enero de 1902, la situación de los mismo no mejoró: primero, porque los municipios, encargados hasta ese momento de pagar los sueldos, tardaron muchos años en abonar la deuda y, por otro lado, porque se mantenían las grandes diferencias salariales entre el ámbito rural y el urbano establecidas por la Ley Moyano (1857) hasta que el Real Decreto de 25 de agosto de 1911 prescribe literalmente que "las escuelas nacionales de primera enseñanza no darán, en lo sucesivo, derecho a sueldo alguno a los Maestros que las desempeñen, en atención a que éstos disfrutarán el que por su lugar en el Escalafón general del Magisterio les corresponda" (MARTÍN ZÚÑIGA, 1991, p. 272).

En síntesis, donde más se nota la falta de estimación hacia el magisterio es en el bajo nivel retributivo. Evidentemente, éste es el problema de la mayoría 
de los asalariados en la España del primer tercio de siglo, pero el caso del maestro es bastante denigrante, puesto que no sólo gana menos que los funcionarios de su misma categoría, -lo que provocó en 1919 las primeras protestas reivindicativas-, sino que su nivel adquisitivo es incluso inferior al de otros profesionales de menor cualificación. No obstante, lo más triste de todo es la "vergonzosa jerarquización salarial" a la que continúa sometida la plantilla del magisterio nacional: «sólo una pequeñísima élite disfruta de los sueldos más altos de las primeras categorías y la inmensa mayoría ocupa las escalas más bajas del escalafón, con retribuciones que no cubren, tan siquiera, la imperiosa necesidad del estómago» (MARTíN ZÚÑIGA, 1991, p. 281)

Lo más singular sobre el tema es el hecho de que el sueldo del maestro o la maestra se podía ver incrementado por parte de los niños más pudientes (art. 192 de la Ley de 1857), una medida como poco controvertida. Para asegurar el cobro de dichas retribuciones e incorporarlas al sueldo oficial, se arbitran diferentes medidas, una de ellas es que sean los propios ayuntamientos, en vez de las familias, quienes se responsabilicen de ese emolumento (MARTíN ZÚÑIGA, 1991). Por ejemplo, el Ayuntamiento de Montijo en las Actas de sesiones, plenos y comisiones gestoras (sesión del 16 de enero de 1932) recoge, en la partida presupuestaria destinada a instrucción pública, la cantidad de 20.271,75 pesetas distribuidas de la siguiente manera: 12.000 pesetas para la adquisición de mobiliario y material pedagógico para las escuelas de nueva construcción, 1.908 pesetas para pagar los jornales de cada una de las responsables de limpieza y 250 pesetas para el aumento del sueldo del maestro.

En el Diario Republicano, La Región Extremeña, hay un artículo de Joaquín Dicenta (1901) en el que se recoge:

[...] es necesario que el maestro no sea un mendigo que bostece de hambre mientras cumple su oficio con indiferencia pasiva, sino un educador que teniendo aseguras comodidades y sustento, pueda dedicar su voluntad, su saber, su existencia entera, a la formación de generaciones vigorosas e inteligentes. 
En síntesis, al escaso número de escuelas hay que añadir, como comentábamos anteriormente, la precariedad en sus condiciones higiénicaspedagógicas y una plantilla de maestros y maestras escasa y mal remunerada. A modo de ejemplo, en el curso 1925-26, según datos extraídos del Anuario Estadístico de 1925-1926, Badajoz gasta en instrucción primaria 2.071.078,33 pesetas, de las cuales son destinadas para el personal docente 1.832.836,37 pesetas y en material 128.243,34. Si tenemos en cuenta, que en esas mismas fechas en Badajoz había 932 maestros, de los cuales 614 eran maestros nacionales y municipales, (98,4\% nacionales y $1,6 \%$ municipales), obtenemos que los profesores no llegan a un salario mínimo de unas 244 pesetas/mes aproximadamente. En cuanto a los centros escolares, había 537 escuelas que eran atendidas por 932 maestros lo que suponía que no había ni dos maestros por escuelas, además la ratio maestro-alumno estaba, con estos datos, en torno a 61 alumnos por cada maestro aproximadamente. A esto hay que unirle las deficientes partidas presupuestarias para el material e instalaciones, pues no llegaban a 300 pesetas por escuela y curso académico.

\section{CONCLUSIÓN}

El objetivo de este trabajo era indagar las causas que impidieron un pleno desarrollo de la alfabetización en la provincia de Badajoz y, no se puede negar, que uno de los factores determinantes del analfabetismo, fue el carácter rural-latifundista de la región. Es decir, la dinámica social y económica que experimentó Extremadura y concretamente la provincia de Badajoz, se caracterizó por tratarse de una región subdesarrollada, donde los altos índices de natalidad, mortandad y emigración, agudizaron el desequilibrio de los diferentes sectores económicos, predominado el sector agrario. García (1985, p. 
991) llega a afirmar, "que en los años treinta del siglo XX la realidad socioeconómica de la región extremeña presenta aún unas características muy semejantes a las que se venían definiendo desde mediados del siglo XIX”. Este desequilibrio era, además, desigual entre los diferentes partidos judiciales dado que no presentaban las mismas características demográficas, socio-económicas, culturales o grado de ruralidad.

Por tanto, estamos ante una región, como sucedió con otras (Andalucía, Canarias, Murcia, etc.), donde la revolución industrial apenas se hizo visible y la economía seguía estando sostenida y dominada por el sector terciario, como hemos mencionado más arriba, por una estructura latifundista al frente de unos cuantos oligarcas que ponían nulo empeño en la escolarización de la masa obrera. A ello se une una clase política, especialmente la municipal, que no propició que la instrucción elemental evolucionara al ritmo adecuado para terminar en un corto plazo con la lacra del analfabetismo, aunque hay que reconocer que, en este periodo, especialmente en los años veinte, se detecta cierta reducción en el problema, aunque es tan lenta que han de pasar muchos años para que esta región se considere plenamente alfabetizada. Probablemente, el hecho de que durante las dos primeras décadas del siglo XX se observen unos valores positivos en cuanto a la disminución del analfabetismo en la comunidad extremeña y concretamente en la provincia de Badajoz, sea porque la administración educativa pasa de estar gestionada por los ayuntamientos a centralizarse en instituciones educativas nacionales.

La media de reducción del analfabetismo en Badajoz en el periodo de estudio se sitúa en torno a diecinueve puntos porcentuales, concretamente veinte puntos para los hombres y diecisiete para las mujeres. No obstante, hubieran sido esperables, en principio, unos resultados más favorables dada la centralización de la educación.

Sin embargo, una de las causas de esa lentitud fue la falta de escuelas, que, aunque aumentaron en número a lo largo de este periodo siguieron siendo insuficientes para atender a toda la población en edad escolar. Además, no 
podemos obviar el pésimo estado de los locales que las acogían y el hacinamiento de las mismas. Por tanto, aunque el número de escuelas fue aumentando no supuso una mejora en los niveles de escolarización.

A esto se le añaden otro problema que obstaculizó y no favoreció el proceso de alfabetización. Nos referimos al absentismo escolar, cuyos porcentajes seguían siendo muy elevados, sobre todo en las zonas rurales y donde la precariedad económica se hacía más patente. A esta situación hay que añadirle, el malestar docente propiciado por un excesivo número de alumnado, unos sueldos deficientes e irregulares y la escasa dotación de medios para la enseñanza, lo que repercutió en una educación deficiente.

Además, hemos comprobado que la escuela era prácticamente la única agencia de alfabetización, por ello la población de seis años, que era la edad con la que se empezaba la enseñanza obligatoria, era prácticamente analfabeta. Pero lo verdaderamente trágico es que en 1930 el 67,4\% de la población entre 6 y 10 años aún no supiera leer y escribir

Otro dato llamativo de estos años es que apenas existían diferencias porcentuales entre niñas y niños de seis a diez años en lo que respecta a la falta de los rudimentos en lectura y escritura, a diferencia de lo que sucedía en las décadas anteriores, y que los datos de escolarización, aunque fueran muy bajos, también eran similares entre ellas y ellos. No obstante, la disparidad entre sexos sigue siendo alta si nos referimos a la población en general. Hemos podido comprobar cómo el 80\% de la población femenina no sabía ni leer ni escribir en 1900, aunque la situación fue mejorando y en 1930 se redujo, redondeando, al $62 \%$, pero la diferencia con los hombres siguió siendo de unos diez puntos.

Por todo lo manifestado, podemos concluir que sí se aprecian síntomas de mejora en los niveles de escolarización y alfabetización de la población extremeña y pacense en particular, donde apenas se encuentran casos de involución o retroceso alfabetizador, lo que supone una mejoría con respecto al siglo anterior, el hecho de que las tasas de analfabetismo continúen siendo tan altas pese a que las autoridades educativas velaron por erradicar los valores 
extremos y los casos de involución, los datos superan con creces la media nacional y aunque, como cabía de esperar, se produce una evolución positiva, algo más en los hombres que en las mujeres, los logros no sólo podemos medirlos en datos cuantitativos, sino también en datos cualitativos, dado que se eliminaron prácticamente las desigualdades entre los territorios y los valores de analfabetismo extremo, pero debido al retraso endémico que sufría la región a nivel económico, social y cultural, todo hacía prever que aún faltan muchos años para que se alcanzara la plena escolarización y alfabetización.

\section{REFERENCIAS}

ARAGONÉS, Antonio Bartolomé. La enseñanza primaria en Olivenza. 19 mayo 1928. En: Correo Extremeño, Año XXV, n. 7545, p. 3. Disponible en: https://prensahistorica.mcu.es/en/catalogo_imagenes/grupo.do?path=203548 4\&posicion=3\&presentacion=pagina. Acceso en: 9 nov. 2019.

BAUMEISTER, Martín. Campesinos sin tierras. Supervivencia y resistencia en Extremadura (1880-1923). Madrid: Ministerio de Agricultura, Pesca y Alimentación, 1997.

BELLO TROMPETA, Luis. Viaje por las escuelas de España.

Extremadura. Madrid: Espasa-Calpe, 1927.

BOHOYO VELÁZQUEZ, Isidoro. Situación socioeconómica y condiciones de vida en la provincia de Badajoz (1880-1902). Badajoz: Editorial Universitas, 1984.

BORRÁS LLOP, José María. Historia de la infancia en la España Contemporánea, 1834-1936. Madrid: Ministerio de Trabajo y Asustos Sociales. Fundación Germán Sánchez Ruipérez, 1996.

CORTÉS CORTÉS, Fernando. La escuela en la Extremadura meridional del siglo XIX. Badajoz: Servicio de publicaciones de la Diputación de Badajoz, 2016.

CORTÉS CORTÉS, Fernando. La instrucción primaria extremeña en el tránsito del siglo XIX al XX. Revista de Estudios Extremeños, v. 54, n. 3, p. 877940, 1998. 
DICENTA, Joaquín. Hacer hombres. 14 agost. 1901. En: La Región

Extremeña, Diario Republicano, Año XXXVIII, 4.470. Disponible en:

https://prensahistorica.mcu.es/es/catalogo_imagenes/grupo.do?path=202058 9\&presentacion $=$ pagina\&posicion $=1 \&$ registrardownload=0. Acceso en: 9 mayo 2019.

DOMÍNGUEZ RODRÍGUEZ, Emilia. Política educativa en el siglo XX y su incidencia en Extremadura. Revista de Estudios Extremeños, v. LXI, n. III, p. 921-984, 2005 .

GABRIEL, Narciso de. Alfabetización, semialfabetización y analfabetismo en España (1860-1991). Revista Complutense de Educación, v. 8, n. 1, p. 217$243,1997$.

GARMENDIA, Joxe; ZABALETA, Iñaki; MURUA, Hilario. Alfabetización en euskera en las escuelas rurales Del País Vasco (1900-1939). Historia y Memoria de la Educación, n. 7, p. 191-233, 2018.

GUEREÑA, Jean-Louis. Estadística escolar y proceso de escolarización (siglos XVIII-XX). En: MORENO MARTÍNEZ, Pedro Luis (coord.). Educación, historia y sociedad: el legado historiográfico de Antonio Viñao. España: Tirant lo Blanch, 2018, p. 315-331.

GUEREÑA, Jean-Louis. La estadística escolar en el siglo XIX. Historia de la Educación. Revista Interunioversitaria, n. 7, p. 137-148, 1988.

HERNÁNDEZ DÍAZ, José María. La escuela rural en la España Del siglo XX. Revista de Educación, Número Extraordinario, p. 113-136, 2000.

Instituto Nacional de Estadística. Anuarios Estadísticos.

JARAÍZ CABANILLAS, Francisco Javier; PÉREZ PAREJO, Ramón; SOTO VÁZQUEZ, José; GUTIÉRREZ GALLEGO, José Antonio. Atlas del analfabetismo en Extremadura durante la segunda mitad del siglo XIX. Madrid: Cultiva Libros, 2017.

JARAÍZ CABANILLAS, Francisco Javier; SOTO VÁZQUEZ, José; GUTIÉRREZ GALLEGO, José Antonio; PÉREZ PAREJO, Ramón; DOMÍNGUEZ MORENO, Guadalupe. Atlas del analfabetismo en Extremadura (1900-1940). Madrid: Dykinson S. L., 2019

LIÉBANA COLLADO, Alfredo. La educación en España en el primer tercio del siglo XX: la situación del analfabetismo y la escolarización. Madrid: Universidad de Mayores Experiencia Recíproca, 2009. Disponible en: 
http://umer.es/wp-content/uploads/2015/o5/n58.pdf. Acceso en: 13 jul. 2020.

LUZURIAGA, Lorenzo. El analfabetismo en España. Madrid: J. Cosano. Museo Pedagógico Nacional, 1919.

MARTÍN ZÚÑIGA, Francisco; SANCHIDRIÁN BLANCO, Carmen. School spaces and school hygiene: two issues not always together (Spain, 1857-1931).

En: ISCHE 38 EDUCATION AND THE BODY. Chicago, 2016.

MARTÍN ZÚÑIGA, Francisco. Categoría y sueldos de los maestros nacionales en el primer tercio del siglo XX. Historia de la Educación - Revista

Interuniversitaria, n. 10, p. 271-282, 1991.

MARTÍN ZÚÑIGA, Francisco. La enseñanza primaria en Málaga durante el reinado de Alfonso XIII (1902-1931). Historia de un retraso endémico. Málaga: Diputación Provincial de Málaga, 1993.

MARTÍN ZÚÑIGA, Francisco. Origen, desarrollo y consecuencias del analfabetismo en el primer tercio del siglo XX. Málaga: Universidad de Málaga, 1992

MARTÍN ZUÑIGA, Francisco; SANCHIDRIÁN BLANCO, Carmen. Edificios y materiales de las escuelas públicas malagueñas (1874-1931), En: XV CONGRESO NACIONAL Y V IBEROAMERICANO DE PEDAGOGÍA Entre generaciones: educación, herencia y promesas, Burgos, 2012.

MOLINO, Sergio de. La España vacía. Viaje por un país que nunca fue. Madrid: Turner Noema, 2016.

REDONDO CASTRO, Cristina. La segunda enseñanza pública en Extremadura (1900-1936), 2018, 440 f. Tesis (Doctorado en Educación) Programa de Doctorado em educación y Comunicación Social, Universidad de Málaga, 2018.

REDONDO CASTRO, Cristina; GRANA GIL, Isabel; SANCHIDRIÁN BLANCO, Carmen. La incorporación de las mujeres a la segunda enseñanza pública en Extremadura (1900-1936). Historia de la Educación - Revista Interuniversitaria, n. 32, p. 277-300, 2017.

REY VELASCO, Fermín. El movimiento obrero en Extremadura entre los siglos XIX y XX. Revista de Estudios Extremeños, v. LIV, n. 2, p. 675-700, 1998.

RODILLO CORDERO, Francisco Javier. Datos para la historia escolar de Extremadura. Mérida: Editorial Regional de Extremadura, 1998. 
RODRÍGUEZ BOOTELLO, Joaquín. Notas de instrucción pública. 1 mayo 1928. En: Correo Extremeño, Año XXV, 7530, p. 4. Disponible en: https://prensahistorica.mcu.es/es/consulta/registro.do?id=10002035468. Acceso en: 6 mayo 2019.

SAMANIEGO BONEU, Mercedes. El problema del analfabetismo en España (1900-1930). Hispania, n. 124, p. 375-400, 1973.

SAMANIEGO BONEU, Mercedes. El problema escolar en Extremadura de 1900 a 1936. Revista de Estudios Extremeños, v. 32, n. 2, p. 281-291, 1976.

TIANA, Alejandro. Educación obligatoria, asistencia escolar y trabajo infantil en España en el primer tercio del siglo XX. Historia de la Educación, n. 6, p. 43-59, 1987.

VILANOVA RIBAS, Mercedes; MORENO JULIÁ, Xavier (1992). Atlas de la evolución del analfabetismo en España de 1887 a 1981. Madrid, CIDE, 1992.

VIÑAO FRAGO, Antonio. Alfabetización y escolarización. En: DELGADO, Buenaventura (coord.). Historia de la Educación en España y América, v. III. Madrid: SM/Morata, 1993. p. 389-396.

VIÑAO FRAGO, Antonio. Del analfabetismo a la alfabetización: análisis de una mutación antropológica e historiográfica. Historia de la Educación Revista Interuniversitaria, n. 3, p. 151-189, 1984.

VIÑAO FRAGO, Antonio. Escolarización y alfabetización. En: MONÉS, Jordi (coord.). La educación en el siglo XX, p. 19-35, 2006.

VIÑAO FRAGO, Antonio. La alfabetización en España: un proceso cambiante en un mudo multiforme. En: MORENO MARTÍNEZ, Pedro Luis; NAVARRO GARCÍA, Clotilde (coord.). Perspectivas históricas de la educación de personas adultas, v. 3, n. 1, p. 5-19, 2009. Universidad de Salamanca.

Disponible en:

http://campus.usal.es/ efora/efora_03/articulos_efora_03/n3_01_vinao.pdf. Acceso en: 9 nov. 2014.

VIÑAO FRAGO, Antonio. Leer y escribir: historia de dos prácticas culturales. Madrid: Fundación Educación, Voces y Vuelos, 1999.

VIÑAO FRAGO, Antonio. Tiempos escolares, tiempos sociales. Barcelona: Ariel Practicum, 1998. 
CRISTINA REDONDO CASTRO es Licenciada en Pedagogía y Doctora en Educación, ambas, por la Universidad de Málaga. Profesora Asociada en el Departamento de Teoría e Historia de la Educación y MIDE de la Universidad de Málaga. Sus principales líneas de investigación incluyen analfabetismo y primera enseñanza, la segunda enseñanza, la historia de la educación de las mujeres, política y legislación educativa y depuración franquista del profesorado.

E-mail: cristinaredondo@uma.es

(b) http://orcid.org/0000-0001-9841-7311

FRANCISCO MARTIN ZÚÑIGA es Profesor Titular de Teoría e Historia de la Educación de la Universidad de Málaga. Sus principales líneas de investigación incluyen analfabetismo e historia de la enseñanza primaria, historia de la educación de las personas adultas, depuración franquista del profesorado y control iglesia-estado de la educación. Entre sus publicaciones relacionadas con el analfabetismo e instrucción primaria destacan Origen, desarrollo y consecuencias del analfabetismo en el primer tercio del siglo XX y La enseñanza primaria en Málaga durante el reinado de Alfonso XIII. Un atraso endémico.

E-mail: fmartinz@uma.es

(i) http://orcid.org/0000-0002-9803-8845

Recebido em: 17 de setembro de 2020

Aprovado em: 28 de janeiro de 2021

Editora responsável: Chris Ramil 